Une simple carte des cours d'eau du ressort de la Société indiquant la situation exacte des zones de frayères pour les principaux poissons devrait ètre dressée à cet eflet par les membres compétents fins observateurs, ct faire partic du dossier de chaque Société. On devrait même l'exiger de toute Société dè Pèche légalement constituée, désirant obtenir dẹs avantages de l'Etat.

Donc, d'abord, surveilliance et protcction des frayères dûment repérées wi moment, de la fraie dans tous les cours d'eau où les poissons, et particulièrement la Truite, peuvent ('ncore sc reproduire naturellement, et, a'autre parl, déversements rationnels d'alevins dans les cours d'eau appauvris, naturellement dépourvus de frayères ou mutilés par l'industrie. Tels sont les deux moyens à mettre en ocuvre par les Sociétés et par tous ceux qui ont lie souci d'assurer le rendement maximum de nos eaux.

La parole persuasive des Présidents ne saura jamais trop s'exercer stir les membres de leur Socićté pour en montrer l'importance, l'intérêt et la nécessité. Et les pêcheurs avisés, éclairés désormais sur le rôle respectif, l'efficacité et les résultats de ces deux mesures fondamentales : protection des frayères et repeuplement, devront s'efforcer de les appliquer à bon escient, en n'oubliant pas quc, pour les cours d'eau convenablement peu. plés qui conservent des frayères accessibles et fonctionnelles, une protection et une surveillance de quelques semaines de ces zones reproductrices suffiront le plus souvent à assurer le repeuplement maximum eî naturel de la rivière en sujets d'élite, c'est-à-dire à leur donner toute satisfactiun et agrément pour le plus grand bien du pays.

Une Société de Pêche qui protège ses frayères lorsqu'elle a le bonheur d'en posséder, qui défend ses eaux contre les empoisonneurs patentés ou non, qui déverse régulièrement à bon escient des alevins dans les cours "'eau dépeuplés privés de frayères ou barrés par l'industrie, est une Société qui remplit ses devoirs au mieux de ses intérêts et de ses aspira-. tions, mérita la confiance et la reconnaissance de tous et, en conséquence, l'appui des pouvoirs publics.

\title{
LE POISSON ROUGE
}

\author{
Par M. Marcel DAGRY
}

Le Poisson rouge ou Carassin doré est originaire d'Asie. On le trouve pius particulièrement en Chine où les habitants, passés maîtres en l'art de la déformation, en font ces monstres si élégants et si décoratifs que sont les Queues de voile et lès Télescopes.

Actuellemnt, cette variété est acclimatée dans nos pays, vit très bien dans les étangs et les pièces d'eau et s'y reproduit à merveille. Malheureusement, elle ne prend en général sa coloration qu'à l'âge de 2 et 3 ans, 
fuand elle atteint ı. à tó centimètres de longueur ; jusqu'à cette taille, les l'oissons sont d'un gris terne et n'ont aucune valeur marchande.

('’est en Italie, principalement dans lá région de Bologne, que se fait iclevagge intensif du Poisson rouge. Les eaux de cette région, ainsi qu'un raitement spécial, permettent de lui donner un accroissement el rune colloration rapides, de sorte qu'au bout de quelques mois on obtient des sujets vendables.

11 y a certains établissements oì cette production ou, plutôt, cette labrication, - car il s'agit là d'une véritable industrie, - a pris un très grand ieveloppement. -

Voici, en peu de mots, comment se pratique cette pisciculture spéciale.

Tout d'abord, il y a les bassins de reproduction, alimentés d'eau courante et maintenus à environ $25^{\circ}$ centigrades. Celte température provoque lia ponte qui est naturelle, c'est-à-dire que les femelles déposent leurs crufs, immédiatement lécondés par les mâles, sur les plantes garnissant le fond des réservoirs.

Au bout de queliques jours, les jeunes sont éclos. Avant que les parents ue dévorent leur progéniture, le bassin est vidé entièrement. Un système de grillages permet aux alevins de passer seuls dans un autre bassin aménagé spécialement pour leur croissance. Les reproducteurs sont remis à nouveau, après triage, dans le bassin de fraye. L'eau est alors maintenue à température plus basse ; ce n'est que 3 ou 4 mois après qu'elle sera de nouveau portée à $25^{\circ} \mathrm{C}$. pour obtenir une nouvelle ponte. On provoque, en général, trois pontes par an ; quelquefois quatre. Les mâles sont changés fréquemment, afin d'obtenir le rendement maximum des fcmelles. Ces dernières ne durent jamais plus de 2 ans.

Dans les bassins d'engraissement, protonds de 40 centimètres environ, lies jeunes Poissons rouges sont soumis à un véritable gavage. Leur faculté d'assimilation est, d'ailleurs, stimulée par ce fait que ces bassins reçoivent une eau dont la température est voisine de $30^{\circ}$ à $32^{\circ}$ C., que l'on aère fortement au moyen de cascades. La nourriture est donnée à profusion ct se compose de viande cruc hachée, de graines crues ou cuites, d'Infusoires et de tout ce qui, en général, provoque l'accroissement rapide des Cyprinides.

Après deux mois de cette suralimentaion, une partie des élèves, réservée pour faire par la suite des reproducteurs, est laissée dans le bassin. On conçoit qu'à la cadence de trois à quatre pontes par an, les géniteurs ne puissent procréer longtemps ; il faut donc pourvoir à leur rempla. cement.

Les Poissons non destinés à laire souche passent dans les bassins spéciaux de colloration. Ces derniers, de faible profondeur (environ $40 \mathrm{~cm}$.), dans lesquels aucun abri n'est aménagé, sont exposés au soleil et alimentés cn eau tiède atteignant parfois $40^{\circ} \mathrm{C}$. C'est une dure épreuve pour les malheureux Cyprins : aussi nombreux sont ceux qui ne supportent pas ce supplice. Ajoutez à cela que divers corps sont dissous dans l'eau pour 
activer la mise en coulleur ; il est facile de se rendre compte de l'effort de résistance vitale demandé à ces jeunes Poissons. Mais il faut savoir souffrir pour devenir beau et le martyre, d'ailleurs, n'est pas de longue durée. Au bout d'un mois les victimes, parées de jollies teintes rouges, noires, jaunes, blanches, quittent cet enfer pour la dernière phase de leur fabrication.

Les produits employés pour provoquer ou hâter la coloration se composent, en général, de lan, de fer ct de noix de Galle, mélangés à d'autres substances dont chaque éleveur croit posséder le secret. Les proportions changent d'ailleurs avec la teinte désirée. Le plus difficille est d'obtenir différentes couleurs sur le même sujet : ceci est le chef-d'œuvre de spécia-

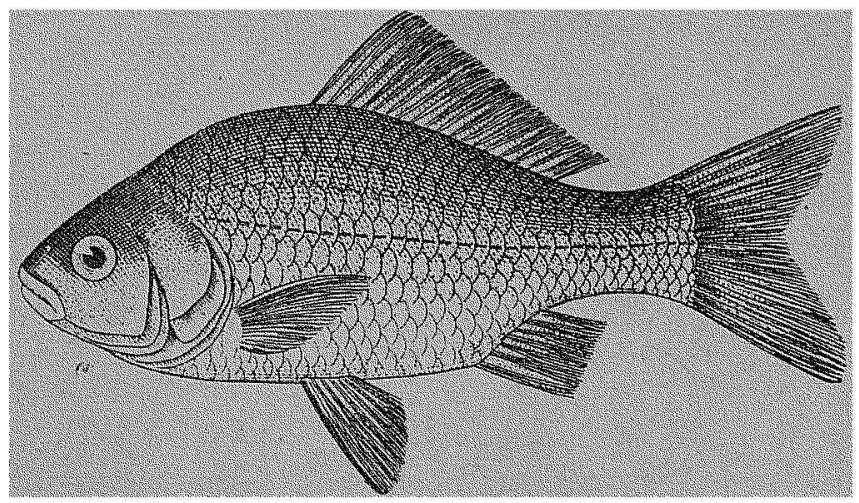

Flg. 33. - Carassin doré ou Poisson rouge.

listes qui, tout en cherchant la juste rémunération de leurs efforts, se proposent aussi un but esthélique, celui de charmer les yeux.

Voici donr nos petites bêtes brillamment parées, mais, les traitements subis leur ont rendu la peau très sensible ; il s'agit, maintenant, de lies remettre en état pour la vente.

Elles sont alors déversées dans des bassins à fond vaseux, boueux ou tourbeux dont l'eau est additionnée de craie et de fer. On arrive, ainsi, à durcir !'épiderme en y fixant définitivement la couleur. Pour cette dernière opéra. tion, la température doit être d'environ $15^{\circ} \mathrm{C}$. Au bout de huit jours, les Poissons sont pêchés, mis à dégorger en eau claire et courante, purgés au sel et ensuite expédiés dans tous les pays, où ils font la joie des enfants $\in$ t lagrément des grandes personnes.

Combien peu de gens, en royant un Poisson rouge évoluer dans leur aquarium, se doutent du travail requis pour arriver à ce résultat ?

Voici, maintenant, notre Cyprin dans sa prison de verre : il y a quelcues précautions à prendre afin de le maintenir en bonne santé.

Pour le Poisson rouge, comme en général pour tous ses congénères d'eau froide vivant en captivité, la température de l'eau n'a qu'une importance secondaire. Ce qui importe, c'est qu'elle ne subisse pas de changements lorsqu'on renouvelle l'eau, et voici pourquoi : 
les Poissons sont des animaux à sang froid, c'est-à-dire que leur sang prend la température du miliẹ dans lequel ils vivent. Mais son réchauffement oll son iefroidisscment doivent se faire lentement, progressiveuent, car, s'il y a brusque variation thermique, aucune réaction ne se produit comme chez les Mammifères et Oiseaux où, justement, la chaleur yopre du sang amorlit (non sans accidents, quelquefois) l'aflet des chanfrements trop rapides de la température extérieure. Chez le Poisson, l'organisme est dépourvu de ce moyen de défense et une saute de températurt occasionne un malaise qui est souvent préjudiciable à la santé.

I.es Cyprins peuvent toutefois supporter, en raison de cette faculté iadaptation thermique qui est une particularité de leur organisme, des variations énormes de température allant de $0^{\circ}$ à $+40^{\circ} \mathrm{C}$. à la seule condilion qu’elles soient lentes comme clles le sont en général dans les eaux naturelles. En aquarium, lorsque l'écart dépasse $7^{\circ}$ à $8^{\circ}$ C. et est brutal, !es Poissons ne peuvent réagir et umc sorte de congestion les éprouve. S'ils ne succombent pas de suite, on les voit, au bout de quelques jours, desrendre inertes au fond de l'aquárium, ne plus manger et la mort vient, linte mais sûre. Aucun soin ne pourra sauver un sujet qui se trouvera lans ces conditions.

Il faut aussi songer à alimenter le captif : Beaucoup de personnes disent : "pour conserver les Poissons, il ne faut pas les nourrir ". D'autres affirment qu'ils se nourrissent de ce qu'ils trouvent dans l'eau. C'est possible à condition de né pas avoir grand appétit, si cette eau est riche en menus organismes. Mais, dans les grandes villles, elle est javellisée, donc lort peu propice au développement des Infusoires dont le Poisson, à la rigueur, se contente.

Dans une mare ou un étang, il n’en est pas de même, les insectes y pulfitlent foumissant au Poisson une nourriture de choix.

Par rapport à sa grandeur, le Poisson mange énormément. En aquarium, où l'espace et l'oxygène lui sont mesurés, il faut, si l'on veut le wnserver, le rationner en ne lui domnant que le strict nécessaire. Y gaver l'hôte est voulọir sa perte, à moins qu'une installation parfaite, donstant de l'oxygène en quantité, ne lui permette de digérer tranquillement sins être incommodé.

Dans le commerce, des aliments sont vendus qui, sous un volume restreint, nourrissent hien le Poisson. Certaines de ces poudres sont très bonnes, d'autres moins. Dans tous les cas, il faudra on doriner très peu, on se tenant aux indications données par le fournisseur, si celui-ci est consciencicux. Toulefois, il sera bon d'alterner cette nourriture, soit avec lies petits vers de vase, soit avec un peu de viande crue hachée, dont les poissons sont très friands. Cela leur donnera les vitamines nécessaires.

In autre point essentiel! est de ne pas surcharger la population de l'aquarium. On dit communémont qu'il faut, à un petit Poisson, un litre d'eau par jour : cela est vrai quand le sujet est réellement petil, mais cette quanlité doit souvent être doublée et même triplée. 
Le maximum de confort est assuré aux élèves en mettant dans leur.prịson quelques plantes aquatiques spéciales, telles que l'Elodea, la Fontinalis, la Vallisneria, etc... Elles donnent à l'eau uu supplérnent d'oxvgène dont les Cyprins ne se plaindront jamais.

Ajoutons qu'il ne faut jamais prendre les Poissons à la main : Jépuisctte est de rigueur pour lles sortir de l'aquarium lọsquè l'on nettoiera ce rernier.

De temps en temps, une fois par quinzaine environ, il sera bon diminerger les Poissons pendant une dizaine de minutes dans de l'eau légèrément salée. Cette médication dégage l'inteslin et ils ne s'en porteroht que mieux.

Les renseignements qui viennent d’ètre donnés permettront aux nombreux amateurs de Poissons rouges de les conserver en bonne santé dans leurs aquariums.

Disons toutefois, en terminant, que s'il est indispensable de connaître ces principes essentiels, e'est seulement en soignant soi-même-ces jolies petites bêtes que l'on se rend vraiment comple de ieurs désirs et de.leur's besoins.

\section{LA SITUATION AGTUELLE DE LA GARPIGULTURE EN POLOGNE.}

\section{Par M. l'Ingénieur EdouARD DE RUDNO-RÜDZINSKI}

Directeur de l'exploitation piscicole d'Osiek (Pologne).

La carpiculture s'est très fortement développée en Pologne depuis ll'indépendance du pays, grâce au prix élevé des Poissons et à l'exclusion des terrains submergés de la réforme agraire. De rouvelles exploitations ont été organisées, celles existant déjà très intensément améliorées.

Les statistiques des nouveaux étangs n'étant pas encore complètement établies, il nous est impossible de connaître exactement la surface de ceux de la Pologne. Le professeur Strafe l'évaluait, en r926, à 6o.ooo Hectares ; elie a dû atteindre, depuis, jusqu'à $7^{5}$.00) Heclares.

Bien que lia consommation des Poissons d'eau douce fût assez basse, (cnviron 750 grammes par habitant et par an), la production du pays était Insuffisante; jusqu'à i'année dernière on importait encore à peu près vingt-cinq pour cent de cette production.

La baisse du prix des Poissons, provoquée par de sérieuses importations, a commencé au printemps de rg3r, si bien que les réserves du pays furent álors vendues à des prix inférieurs à ceux de l'automne I93o. Depuis cette epoque, la baisse, aggravée par la crise mondiale, a été continue bien que les importations aient cessé.

Le prix de la viande, diminuant, a entraîné aussi celui du Poisson et ia 